\title{
INVESTIGACIONES
}

\section{La comprensión del cerebro y la educación de personas jóvenes y adultas}

\author{
Understanding the brain and the education \\ of adult and young people
}

\author{
María Eugenia Letelier Gálvez ${ }^{a}$ \\ ${ }^{a}$ Universidad Metropolitana de Ciencias de la Educación (UMCE). \\ m_eugenia.letelier@umce.cl
}

\section{RESUMEN}

El artículo indaga en los aportes de la neurociencia cognitiva a la educación de personas jóvenes y adultas. Los avances en la comprensión del cerebro abren contribuciones nuevas en el ámbito educativo, el deseo de explorar en estas contribuciones sustenta este texto basado en el análisis de un proceso de investigación participativa desarrollado con docentes de educación de personas jóvenes y adultas. El estudio aborda temas destacados en neurociencia como son ambiente de aprendizaje, memoria y recuerdo, motivación y emociones, ansiedad y estrés, sistema atencional y organización del espacio relacionándolo con la diversidad y complejidad que enfrenta la educación de personas jóvenes y adultas. El texto concluye que para avanzar en la comprensión del cerebro se requiere una visión interdisciplinaria que articule las ciencias biológicas con los avances desde la sociología, psicología y antropología incorporando como perspectiva central la reflexión pedagógica de las y los educadores.

Palabras claves: educación, educación de personas jóvenes y adultos, neurociencia cognitiva.

\begin{abstract}
The paper explores cognitive neuroscience contributions to the education of adult and young people. Advances in brain understanding make new contributions in the educational field, the will of exploring these supports this text based on the analysis of a participatory research process developed with young and adult people teachers. The study addresses outstanding topics in neuroscience such as learning environment, memory and remembering, motivation and emotions, anxiety and stress, attention system and space organization as they relate to the diversity and complexity the education of adult and young people faces nowadays. The text concludes that advancing in brain understanding requires an interdisciplinary vision which articulates biological sciences with advances from sociology, psychology, and anthropology incorporating as a central perspective the educators'teaching reflection.

Key words: education, education of adult and young people, cognitive neuroscience.
\end{abstract}




\section{INTRODUCCIÓN}

A partir de 1990 se inicia un movimiento internacional que asume el aprendizaje a lo largo de la vida como un principio rector para la educación del siglo $\mathrm{XXI}^{1}$. Este enfoque reconoce la capacidad del ser humano de aprender siempre en los diferentes espacios en que desarrolla su vida y no únicamente en la edad escolar y a través de la educación formal.

La emergencia de este enfoque se sustenta en la vertiginosidad de los cambios y en el desafío que supone vivir en una sociedad que se torna cada vez más compleja. Las aceleradas transformaciones tecnológicos, la disponibilidad creciente en cuanto a acceso y producción de información, los cambios demográficos con el aumento de la esperanza de vida exigen que cada persona deba prepararse constantemente para desenvolverse en un entorno que demanda nuevas competencias, en todos los ámbitos y en todas las edades.

Los nuevos enfoques dialogan con los avances en la comprensión del cerebro. En los 90 las ciencias biológicas se introducen en el campo de la educación, espacio habitual de otras disciplinas de las ciencias sociales como la sociología, psicología o antropología. Conferencias de expertos convocados por la Organización para la Cooperación y el Desarrollo Económico (OECD) marcan un hito al tejer en la comunidad internacional un puente entre "las nuevas ciencias del cerebro y la educación". Las investigaciones de la neurociencia y la difusión de sus avances en la comunidad internacional (OECD, 2003) aportan nuevas evidencias para fundamentar el aprendizaje a lo largo de la vida. Los conceptos de plasticidad y vitalidad cognoscitiva en los adultos son identificados como las claves para asegurar el aprendizaje durante toda la vida. Las investigaciones de los neurocientíficos confirman la capacidad del ser humano para aprender siempre, a lo largo y a lo ancho de su vida.

Esta perspectiva del aprendizaje cobra especial relevancia para la modalidad de educación de personas jóvenes y adultas. Convocada por la UNESCO, se celebra cada diez años la Conferencia Internacional de Educación de Adultos (CONFINTEA), la última, realizada el año 2009 marca la transición hacia este nuevo enfoque "De la alfabetización al aprendizaje a lo largo de la vida". La declaración final suscrita por los países señala "El aprendizaje a lo largo de toda la vida cumple una función crítica en la manera de abordar los problemas y los retos mundiales de la educación. El aprendizaje a lo largo de toda la vida, de la cuna a la tumba, es un marco filosófico y conceptual, y un principio organizativo de todas las formas de educación" (UNESCO, 2009, p. 3).

Si se asume que el enfoque de aprendizaje a lo largo de la vida otorga una nueva perspectiva a la educación de personas jóvenes y adultas y que las avances en el conocimiento del cerebro son especialmente relevantes para esta modalidad, es dable preguntarse ¿en qué aspectos los aportes provenientes de la neurociencia cognitiva pueden ayudar a comprender mejor las condiciones necesarias para el logro de los aprendizajes y diseñar una oferta pertinente en esta modalidad educativa?

Para avanzar en la respuesta se analizó información procedente de un trabajo desarrollado con docentes de educación de personas jóvenes y adultas en una comuna de

Desde el año 90, cada 15 años se celebran las Conferencias Internacionales del Movimiento de Educación Para Todos, la primera conferencia en 1990 en Jomtién (Tailandia), la segunda en 2005 en Dakar (Senegal) y la Tercera en Incheón (Corea) en 2015, la articulación de las conferencias la impulsa la UNESCO a nivel mundial. El mismo enfoque se asume en los Objetivos de Desarrollo Sostenibles que marca la Agenda 2030 acodada por la Asamblea General de las Naciones Unidas en el año 2015, para los próximos 15 años. 
Santiago de Chile. Durante cinco meses, a través de una metodología activo-participativa, se trabajó con cuarenta y cinco docentes pertenecientes a cinco establecimientos distintos. En el período se implementaron seis talleres en los que participaron todos los docentes; cada sesión fue precedida por trabajos individuales y/o grupales sobre la base de un breve texto y una pauta que guiaba la conversación. En las sesiones se abordaron temas claves relacionadas con el contexto y características específicas de las y los estudiantes; la motivación para ingresar y permanecer; las dificultades y potencialidades de aprendizaje y los desafíos pedagógicos. Las reflexiones de los docentes se capturaron de manera textual y fueron analizadas teniendo como marco de referencia hallazgos provenientes de estudios en neurociencia ${ }^{2}$.

\section{NEUROCIENCIA Y APRENDIZAJE A LO LARGO DE LA VIDA}

A principios del siglo XIX un joven alemán que vivió su infancia y adolescencia encerrado y aislado en una cueva, que no conocía el lenguaje ni tenía capacidad de relacionarse con los demás, es abandonado en un pequeño pueblo alemán convirtiéndose en el "atractivo" de la comunidad científica de la época. Kaspar Hausen, con 16 o 17 años, aprendió a hablar, a leer y escribir y adquirió conocimientos de filosofía, latín, música y ciencias. A los cinco años de haber sido encontrado murió víctima de un asesinato. Aunque su muerte se transformó en un nuevo enigma, lo cierto es que la trepanación de su cerebro fue motivo de gran interés entre los científicos ${ }^{3}$. El interés por conocer cómo funciona el cerebro no es nuevo, historias como Kaspar Hausen, en pleno apogeo del positivismo, pueden considerarse las predecesoras de la neurociencia cognitiva en la modernidad.

Hacia finales del siglo XIX, el desarrollo científico permitió un hallazgo fundamental en la comprensión de la anatomía del sistema nervioso, las investigaciones de Ramón y Cajal (1852-1934) mostraron que el sistema nervioso está formado de células independientes. Estas investigaciones pioneras facilitaron la identificación de las dendritas como el medio en que las neuronas se conectan entre sí. Posteriormente, se avanzó en comprender la presencia de neurotransmisores como sustancias que ayudan al intercambio de información química entre las células, se introduce el término "sinapsis" para identificar la comunicación entre las neuronas.

A pesar de los avances en la comprensión del cerebro, hasta mediados del siglo pasado, la concepción dominante sostenía que las neuronas solo se generan en la vida prenatal y en los primeros años de la niñez. Fue recién, a partir de los años 90 cuando estudios realizados por Fernando Nottebohm ${ }^{4}$, entregan las pruebas definitivas que la neurogénesis; es decir, la capacidad de producir nuevas neuronas ocurre también en el cerebro adulto, ocurre durante toda la vida.

Para presentar la información se optó por codificar Prof. (profesores) E. Educativo (establecimiento educativo de pertenencia).

Existen numerosos libros y artículos de opinión que especulan sobre la vida y el asesinato. Entre ellos destaca la película de Werner Herzog "El enigma de Kaspar Hausen” (1974).

$4 \quad$ La participación de la neurogénesis en el aprendizaje se demostró por primera vez en el cerebro de las aves por Fernando Nottebohm (1989). 
La capacidad del cerebro de mantenerse flexible, alerta, sensible y orientado a la búsqueda de soluciones se debe a su capacidad de plasticidad durante toda la vida. En cierto momento, los neurocientíficos pensaban que sólo los cerebros de los infantes eran plásticos (...) sin embargo, datos de primates no humanos y de humanos, descubiertos en las dos décadas pasadas, han confirmado que el cerebro mantiene su plasticidad durante toda la vida... (OECD, 2003, p. 96).

Estudios posteriores además de corroborar que el ser humano puede aprender a lo largo de toda la vida, muestran que el aprendizaje es capaz de transformar el cerebro,

La plasticidad del encéfalo no constituye una estructura estática, sino que se adapta sin cesar a las condiciones de su entorno, es decir, es plástico. La plasticidad se manifiesta, en primer lugar, porque las conexiones entre neuronas (las sinapsis) reaccionan con mayor sensibilidad. Por otro lado, pueden dar lugar a conexiones completamente nuevas. Estos mecanismos configuran la base del aprendizaje" (Scholz, J. y M. Klein, 2011, p. 12).

Profundizando, nuevas investigaciones muestran que además de su plasticidad y la capacidad de producir neurogénesis en la vida adulta, las demandas de nuevas habilidades inciden también en los procesos bioquímicos; es decir, nuevas exigencias del medio también producen cambios en el cerebro.

El perfeccionamiento de una nueva habilidad acarrea cambios bioquímicos en las neuronas, lo que da lugar a transmisiones más fuertes o más débiles a través de sinapsis. Este tipo de plasticidad básica persiste durante toda la vida. Siempre puede aprenderse algo nuevo (Hensch, 2016, p. 47).

Se puede afirmar que durante los últimos 100 años las investigaciones han avanzado con tanta celeridad que ha permitido a los científicos transitar desde una comprensión global del cerebro hacia una comprensión del funcionamiento de las neuronas y de la interrelación entre ellas ${ }^{5}$. A pesar de estos avances, aún falta mucho camino por recorrer y, seguramente, será difícil explicar con exactitud las múltiples funciones e interrelaciones que permitan comprender la manera cómo funciona el cerebro.

La evidencia científica puede tardar, no obstante, es desafiante intentar una aproximación que abra puentes entre los avances de los neurocientíficos y la experiencia y reflexiones provenientes de la pedagogía y de las ciencias sociales.

\section{APORTES DE LA NEUROCIENCIA: REFLEXIONES CON DOCENTES DE EDUCACIÓN DE PERSONAS JÓVENES Y ADULTAS}

En líneas generales, los fundamentos proporcionados por las ciencias sociales son conocidos por los educadores de personas jóvenes y adultas. La sociología contribuye desde una mirada crítica de la sociedad explicando la inequidad social y la desigual distribución de

A fines del 2018 se publicó en la prensa los avances de un grupo de científicos que han descubierto un nuevo tipo de neurona solo presente en los humanos "escaramujos" Recuperado en https://www.bbc.com/mundo/amp/noticias-45330141 
las oportunidades que tienen las personas en el sistema educativo; la antropología desde un enfoque cultural proporciona elementos para comprender los marcos interpretativos que configuran la identidad y diversidad de las personas que participan en la modalidad; la psicología proporciona fundamentos en relación a ciclos de vida, variables socio-afectivas, autoestima y las motivaciones y emociones.

La neurociencia es una contribución relativamente nueva que causa muchas expectativas en tanto respuesta a los múltiples desafíos de la labor docente. Se puede decir que, hasta hace poco, las ciencias biológicas y la pedagogía seguían caminos independientes. Asumiendo la necesidad de visibilizar temáticas comunes entre las diferentes disciplinas y partiendo de las reflexiones de los docentes de educación de adultos, nos proponemos relevar aspectos específicos para incentivar el diálogo entre las ciencias sociales, la pedagogía y la neurociencia.

Para abordar los aportes de la neurociencia al campo de la educación de personas jóvenes y adultas se distinguen cinco temas que buscan articular ciencias biológicas con las ciencias sociales desde la reflexión de las y los educadores: ambiente enriquecido, memoria y recuerdo, ansiedad y estrés, espacio, sistema atencional.

\subsection{AMBIENTE ENRIQUECIDO}

Desde la perspectiva de la neurociencia el ambiente es fundamental para lograr el aprendizaje, un ambiente enriquecido aumenta la neurogénesis en el hipocampo. La ciencia experimental, basada en investigación con roedores, mostró cómo un grupo de roedores en una jaula con varios objetos y acompañado de otros roedores logró un mejor desempeño que ratones aislados situados en un ambiente empobrecido. En gran medida, este experimento, ha sido la base para sostener que los ambientes enriquecidos estimulan de mejor manera la producción de sinapsis y la disposición al aprendizaje.

Las ciencias biológicas identifican períodos críticos, sobre todo en la infancia y juventud, para el desarrollo de estructuras relacionadas a determinadas funciones, si durante estos períodos las influencias ambientales son pobres, estas funciones no se desarrollarán de forma adecuada. Las investigaciones refieren a "ventanas de oportunidades" identificando períodos críticos en los cuáles el potencial de aprendizaje es mayor.

Desde la práctica y reflexión pedagógica los docentes expresan:

La mayoría de los estudiantes provienen de situaciones socioeconómicas y familiares altamente vulnerables, con una infancia y adolescencia con escasas oportunidades, muchos estudiantes tienen vergüenza por haber abandonado sus estudios, porque dejaron la escuela y también por la manera en que viven, la precariedad de su entorno, se ocultan ante los profesores y ante sus compañeros.

(Prof. 1 E. Educativo 1)

El conocimiento que traen es bajo, tienen un lenguaje empobrecido que obstaculiza adquirir nuevos aprendizajes, el entorno de vida resulta poco estimulante, niveles muy bajos de alfabetismo repercuten en la valoración social del aprendizaje, el manejo del vocabulario es muy restrictivo. Lo que ven es lo que ofrece la cultura en que están insertos y con frecuencia viven en un entorno deprimido, con muy pocos incentivos, para muchos la sobrevivencia es una exigencia diaria. (Prof. 2 E. Educativo 2) 
Estas características relacionadas tanto con oportunidades de aprendizaje en la infancia como con el entorno de vida actual podrían llevar fácilmente a un cierto determinismo que limite el umbral de aprendizaje a lo "posible" con "este tipo de estudiantes". ¿En qué medida será factible lograr el aprendizaje aun cuando la biografía y trayectoria de vida de las personas ocurran en un entorno deprimido?

De acuerdo con los hallazgos científicos una estrategia para restaurar la plasticidad consistiría en eliminar las trabas que impiden el reinicio de los períodos críticos. Uno de los límites de la plasticidad reside en una red de moléculas llamada red perineuronal (...) los frenos a la plasticidad desaparecen cuando estas moléculas son digeridas por enzimas (Basado en Hensch, 2016).

Investigaciones que vinculan la neurociencia y educación han buscado responder cuánto influye el ambiente en el aprendizaje. Entre los especialistas se destaca que esta relación no es unívoca y coloca en relación autoimagen, motivaciones y clima. "Lo que sabemos es que el aprendizaje exitoso es más probable si el que aprende a) tiene una alta confianza y una buena autoestima b) está fuertemente motivado para aprender y c) es capaz de aprender en un medio caracterizado por "retos altos" asociado con "pocas amenazas" (OECD, 2003).

Los docentes expresan que los ambientes de aprendizaje se enriquecen construyendo experiencias positivas, se puede decir que la estimulación va abriendo potencial de acción en directa relación con el trabajo que los docentes incentivan al hacer del entorno un espacio grato, con exigencias, pero sin amenazas.

Alprincipio del curso nadie dice nada, de a poco entran en confianza y van interactuando, se escuchan y hacen preguntas y se va generando otra disposición. Es un proceso lento y no todos lo experimentan. Lo interesante es observar que los propios participantes, cuando retoman sus estudios, perciben que su decisión repercute no solo en ellos como personas, sino también en la familia y en otros espacios de inserción social y laboral. Se puede afirmar que la integración a procesos de escolarización y/o de nivelación de estudios tiene un efecto positivo en el entorno y favorece que otras personas aprecien el valor social del aprendizaje. (Prof. 3. E. Educativo 3)

Para lograr encantarles, no sólo con la escuela sino con la familia, con la sociedad, con sus vidas, se debe crear un ambiente sin amenazas, pero que los desafíe, esa es la manera ideal para desplegar las potencialidades de aprendizaje. El espacio cultural que tienen en sus vidas es el colegio, es el ambiente más enriquecido al que pueden tener acceso, aquí ellos tienen que tener la experiencia de la emoción por aprender, descubrir sus talentos. (Prof. 4. E. Educativo 4)

\subsection{MEMORIA Y RECUERDO}

La memoria y el recuerdo son fundamentales en el proceso de aprendizaje. Los avances científicos han permitido transitar desde una explicación de la memoria como una especie de "video o cinta fija que se repite" hacia la memoria como la creación de un cambio persistente en el cerebro mediante un estímulo, es decir, como un proceso y no como una localización fija. 
La memoria es el resultado de un complejo y amplio grupo de interacciones entre estructuras cerebrales y procesos de codificación de la estimulación que nos rodea. Existe una clara colaboración entre sistemas. Uno de ellos se halla implicado de forma directa en los procesos que median la activación emocional y se refleja por los efectos que provoca sobre otros sistemas de memoria, la declarativa (López, 2012, p. 45).

Siguiendo al autor citado, la memoria declarativa corresponde a un tipo de memoria a largo plazo, son aquellos recuerdos que pueden evocarse de manera consciente. Entre estos recuerdos está la memoria de los hechos y de los eventos, esta memoria permite desarrollar procesos cognitivos y metacognitivos.

Para las personas jóvenes y adultas, una dificultad consiste en que estos procesos no han sido suficientemente desarrollados y llegan a la educación media sin contar con conocimientos básicos. Falta desarrollar conceptos de temporalidad y localización espacial, conceptos abstractos como álgebra, incluso en el segundo nivel de educación media, son difíciles de lograr, falta vocabulario básico para la comprensión lectora, dificultades de comprensión lectora básicas para entender un enunciado. (Prof. $1 \mathrm{E}$. Educativo 1)

A su vez es necesario considerar que en las experiencias de vida existen obstáculos cognitivos -convicciones erróneas- que tienen un estatus de verdad en las personas y que bloquean la adquisición de nuevos conocimientos. Las personas adultas requieren de un proceso educativo intencionado y de calidad que sea capaz de ayudarlas a superar esos obstáculos. Estas convicciones erróneas, generalmente, están relacionados con temas como la salud, la electricidad, la alimentación; es decir, con temas de la vida cotidiana en torno a los cuales se han realizado sus propias hipótesis. (Prof. 3. E. Educativo 3)

Fuera del hipocampo y estructuras afines, se localiza la memoria no declarativa, o de procedimiento, que permite realizar las acciones de forma automática, cuyos subsistemas cuentan con un amplio grupo de áreas cerebrales. Las y los docentes vinculan el aprendizaje con la memoria de experiencias anteriores que inciden en la disposición y capacidad de las y los estudiantes.

Asimilan situaciones nuevas con recuerdos, con experiencias previas. Los estudiantes han escuchado muchas veces que no son capaces, algunos tienen experiencias traumáticas, les han dicho que son tontos que no sirven para estudiar. Hay algunos que tienen miedo al profesor, miedo a las materias, miedo a no aprender. La historia de cada uno es muy compleja, portan una identidad deteriorada marcada por miedo a no ser capaces, por los recuerdos de experiencia previas. Tienen una gran sensación de fracaso y miedo, sobre todo en inglés o matemática parten diciendo que no saben nada y se predispone a no aprender. Asimilan situaciones nuevas con recuerdos, a veces, traumáticos, todas son barreras que condicionan. (Prof. 5 E. Educativo 4)

A su vez, estrategias erradas basadas en experiencias pedagógicas previas como repetir sin entender, copiar sin saber, memorizar sin comprender, atender solo a un fragmento de información, suelen ser incorporada por las personas como memoria 
no declarativa que las lleva de manera automática a realizar procedimientos que dificultan el aprendizaje. Con frecuencia personas mayores en su socialización temprana, desarrollaron estrategias de repetición, llegan aquí y buscan memorizar y repetir y eso no les ayudó antes y ahora tampoco. Estudiantes que en su infancia o en su adolescencia no incorporaron habilidades básicas o que las incorporaron de manera errada, luego tienen dificultades para incorporar nuevos aprendizajes. (Prof. 6 E. Educativo 5)

Si pensamos que la memoria no declarativa está relacionada con habilidades que las personas ponen en movimiento de manera más o menos automática, debemos reconocer que las personas adultas traen a los procesos de aprendizaje una "huella" muchas veces difícil de revertir, el desarrollo de la habilidad de leer y escribir constituye un aspecto muy sensible que repercute en las personas a lo largo de su trayectoria. No obstante, como señalan los docentes.

Simultáneamente las personas adultas son portadores de otras habilidades que el sistema educativo y muchas veces la propia sociedad suele no valorar, las personas han desarrollado destrezas que les han permitido desenvolverse en su entorno, las cuales, al ser valoradas, constituyen un buen punto de partida para nuevas adquisiciones cognitivas. (Prof. 5 E. Educativo 4)

\subsection{MOTIVACIÓN Y EMOCIONES}

La motivación está íntimamente ligada con las emociones, de acuerdo con la neurociencia, estas últimas constituyen la forma en que el cerebro evalúa si actuar o no sobre las cosas -aproximarse a ellas si son placenteras y evitarlas si son desagradables-. Por lo tanto, es posible formular la hipótesis de que los sistemas emocionales crean motivación.

Las emociones son una fuente de información fundamental para el aprendizaje. Las emociones desencadenan los cambios químicos que alteran nuestros estados de ánimo y conductas. Las emociones no son independientes de los contextos como los valores que tenemos.

Las emociones se basan en las experiencias vitales, a través de la experiencia se moldean nuestros comportamientos biológicos asociados al miedo, preocupación, sorpresa, alegría, alivio, entre otros. "Debemos abandonar la vieja costumbre de pensar en las emociones como algo siempre irracional o que nada que ver con el modo que pensamos; las emociones son una fuente fundamental de información para el aprendizaje" (Jensen, 2010, p. 103).

Entre los diversos impulsos que motivan a las personas a aprender, incluyendo el deseo de aprobación y reconocimiento, uno de los más poderosos es la emoción de comprender, de saber, aquello que a veces se expresa en forma coloquial "se me iluminó la ampolleta". El cerebro responde de manera excelente, cuando súbitamente hace conexiones y conecta la información que tiene disponible con la nueva que le es propuesta. Esta comprensión profunda "es el placer más intenso que el cerebro puede experimentar, en el contexto del aprendizaje" (OECD, 2003). 
Si existe esta convicción vale la pena preguntarse: ¿Qué es lo que hace que algunos jóvenes y adultos quieran aprender, se comprometan con el aprendizaje, mientras que otros prefieran quedarse al margen?

Hay una motivación externa, porque la sociedad les exige terminar la Educación Media, pero no es la única ni la más importante razón por la cual se quedan y aprenden. Para que se motiven hay que reforzar, señalar los logros, los refuerzos positivos son fundamentales, cuando se sienten reconocidos, cuando se sienten capaces, es cuando se quedan. Si la primera nota es un tres, ya no vuelven, tienen poca tolerancia a la frustración, algunos vienen piteados, vienen igual y por supuesto que nos damos cuenta, pero es mejor que estén a que vayan a la plaza. Es necesario romper con el estigma que traen, hay una experiencia de fracaso que tiene que revertirse, que puedan decir: lo logré. (Prof. 7 E. Educativo 3)

Se debe considerar que el riesgo de deserción es permanente, existe una cultura desertora, es fundamental que ellos se sientan valorados como persona, se sientan parte del proceso o de lo contrario se van. La motivación se consigue cuando tienen logros que pueden ver, por eso los trabajos deben tener objetivos claros y deben ser breves. La motivación tiene que ser clase a clase, que ellos vean sus logros en el proceso, paso a paso. (Prof. 8 E. Educativo 4)

Hay que ponerse metas comunes para el grupo, que vean que hay una meta por alcanzar y que todos la pueden conseguir. Para lograr la motivación efectiva, los estudiantes tienen que alcanzar autonomía, de a poco, se tienen que sentir responsables de su proceso de aprendizaje, son ellos los que tienen que tener ganas de aprender. (Prof. 9 E. Educativo 5)

Un dilema en el proceso es cómo ofrecer una mirada distinta, generar otros referentes, despertar otros intereses. Nosotros tenemos que mostrar otras maneras de ver, otros referentes, el interés no puede basarse solo en lo que ellos ya conocen, ¿cómo hacer para producir un vínculo entre sus motivaciones y los nuevos conocimientos que se quieren lograr? este es un dilema pedagógico. Todos sabemos que la atención se consigue cuando se abordan los temas de manera amena, cercana, con ejemplos de lo que ellos viven. No obstante, si selecciono un poema o un texto que no es de su vida cotidiana, a lo mejor se pueden aburrir, pero debo enseñárselos para abrir otros puentes en su vida. (Prof. 6 E. Educativo 5)

Podemos buscar muchas estrategias para lograr la motivación y la atención, pero a clave siempre está en las emociones, hay que ser artista, creativo para gatillar emociones positivas, que vengan contentos que encuentren un espacio en que se les acoge y que descubran la emoción de aprender. En el proceso, se logra la motivación efectiva cuando los estudiantes vienen a intentar construir su identidad "estoy aquí” no solo con sus profesores, es con sus compañeros, con el grupo, que se le reconozca, que logre sentido de pertenencia que se les reconozca como sujetos de aprendizajes, que se les reconozca y valore sus capacidades y su aporte. (Prof. 3. E. Educativo 3) 
El sistema de recompensa está asociado a una serie de áreas y conexiones nerviosas que contribuyen a proporcionar sensaciones de bienestar. Cuando experimentamos felicidad, se activan unas áreas cerebrales que se comunican entre sí a través de determinados mensajeros químicos.

Un mensajero químico clave es la dopamina, al liberarse, se abre camino a un área del cerebro llamado núcleo accumbens, lo que desencadena la retroalimentación que predice si algo bueno o malo está a punto de suceder. De acuerdo con C. Christine Wolf,

Una estación de encendido central para los mecanismos neuronales que subyacen en este proceso es el núcleo accumbens, un grupo de neuronas situado en la parte inferior del prosencéfalo. Una importante función también la desempeña el septrum, estructura membranosa de células gliales (...) hoy sabemos que tanto el núcleo accumbens como el septum forman parte de un extenso circuito de recompensas: el sistema dopaminérgico. Las neuronas que participan en él emplean el mensajero químico dopamina para la transmisión de las señales en las sinapsis, de ahí su nombre (Wolf, 2013, p. 80).

Las opiniones de los docentes coinciden en las recompensas como clave para lograr la motivación al aprendizaje y esta recompensa, para que sea duradera y efectiva, está basada en la emoción de reconocerse como un legítimo aprendiz.

\subsection{ANSIEDAD Y ESTRÉS}

En la medida que se avanza en la comprensión del funcionamiento del cerebro, se evidencia que la mejora en los procesos cognoscitivos y compartimentales asociados a la neurogénesis adulta están relacionados con la capacidad de las neuronas para integrarse a circuitos ya existentes, en este comportamiento influyen poderosamente la ansiedad y el estrés.

Estudios realizados tanto en humanos como en roedores han permitido avanzar hacia la comprensión de los efectos del estrés sobre la neurogénesis adulta.

A pesar de que el estrés agudo mejora el desempeño del individuo y lo prepara para tener respuestas adaptativas, existe también un efecto negativo. Se ha demostrado que la exposición a diferentes estímulos generadores de estrés disminuye la supervivencia de las células recién creadas, pero no la proliferación, en el giro dentado del hipocampo adulto (...) de esta forma, es posible que el efecto del estrés agudo sobre el proceso de neurogénesis sea dual, aumentando la proliferación y la diferenciación, pero disminuyendo parcialmente la supervivencia de las nuevas células (Zárate et al., 2014, p. 40).

Muchos se estresan con tareas que desde nosotros son simples, por ejemplo, hablar delante de sus compañeros, se sienten inquietos y observados, he visto como algunos se ponen a sudar y se quedan sin voz. Algunas personas mayores que les cuesta mucho la tecnología se sienten presionados por adquirir habilidades que no tienen, se sienten superados por los más jóvenes y se quedan paralizados. (Prof. 10 C. Educativo 4)

Aunque parezca evidente, las investigaciones indican que el adulto aprende mejor cuando está saludable y descansado (Elbers, 2015). Esto tiene importantes repercusiones prácticas, 
pues se sabe que el estrés, definido como una respuesta general biológica, fisiológica y conductual del organismo frente a una posible amenaza, limita el procesamiento de la información. Un sujeto estresado distorsiona, borra y/o simplifica la información, lo que puede perturbar el aprendizaje, un sujeto que no está bien alimentado y no ha descansado tiende a estar más estresado y, como está demostrado, perjudica también el estado de ánimo.

Con frecuencia nuestros estudiantes vienen mal alimentados, vienen de jornadas de trabajo extensas y es difícil lograr la concentración, de alguna manera este es un tiempo para ellos, el resto del día siempre tienen otras obligaciones. Los estudiantes de EPJA han vivido un rompimiento doloroso al dejar la escuela, no han encontrado contención social o familiar, se enfrentan a situaciones de temor, de no ser capaces. Siempre estamos señalándoles que tenemos altas expectativas, que pueden lograrlo, ponemos metas factibles, que valoren el esfuerzo y permita superar la ansiedad y el estrés. (Prof. 11. E. Educativo 1)

Procuramos crear un ambiente agradable, con exigencias, pero sin estrés, en eso favorece que la modalidad no tenga que dar pruebas estandarizadas, nos quita la tensión de tener que preparar, que vivir en función del puntaje de los estudiantes.

Para avanzar en el manejo del estrés y la ansiedad hay un proceso que parece fundamental. La aceptación de sí mismo, conocerse como es, lo que puede aportar, lo que sabe, identificar cuáles son sus talentos. Hay que reconocer las diferencias, algunos, por ejemplo, no les gusta hablar en público, otros son más inquietos, otros observan. Todos tienen maneras distintas de ser. (Prof. 5 E. Educativo 4)

Si vinculamos estas opiniones con investigaciones que asocian la neurogénesis con la capacidad del cerebro de captar señales sensoriales para evocar una experiencia previa, proceso conocido como separación de patrones que permite ordenar nuestros recuerdos y guiar nuestros actos, el reconocimiento de capacidades y potencialidades parece un buen camino para aliviar la ansiedad y el estrés en los procesos de aprendizaje.

\subsection{ESPACIO}

Desde la neurociencia se ha avanzado en identificar lo que se ha dado en denominar un sistema de navegación del hipocampo que cumple una función que va más allá de la manera de desplazarse de un lado a otro.

Aparte de recibir la información sobre la posición, la distancia y la dirección desde la corteza entorrinal medial, el hipocampo crea un recuerdo de lo que hay en ese lugar y de los sucesos que tienen lugar en él. Así pues, el mapa espacial creado por las neuronas de ubicación no contiene información sobre el paradero del animal, sino detalles sobre su experiencia (Moser, May-Britt y Edvard I. Moser, 2016, p. 23).

Este aporte de la neurociencia incorpora elementos de análisis interesantes para comprender mejor a las personas jóvenes y adultas que participan de la modalidad. Habitualmente quienes llegan a la modalidad son personas que viven en espacios reducidos, algunos hacinados, donde no hay separación de espacios para funciones distintas. 
Falta mayor reflexión crítica en torno al espacio que se habita, en el país en que se vive. Es interesante observar como al principio para ellos el espacio de la plaza o el de la cocina es el mismo, les da igual el lugar. No diferencian funciones porque en las casas está todo junto, no hay espacios propios para ellos, por lo mismo cuesta poner límites, cuesta que se regulen, cuesta poner reglas, no saben cómo actuar en el espacio educativo. Pero aquí se van dando cuenta que este espacio es distinto que es un lugar para ellos, que les pertenece y lo respetan. (Prof. $1 \mathrm{C}$. Educativo 1)

Van generando un espacio propio, lo que une a los estudiantes es súper meritorio, porque están aquí y no están en la plaza, entran y se quedan, a veces abúlicos, pero están, se quedan. Hay que repetir las normas, volver a decir lo que ya se les dijo, pero es importante porque va ayudando a conocer los límites y que sepan que estas normas se cumplen. En el espacio educativo los jóvenes buscan volver a sentirse estudiantes, "normalizarse" nuevamente, necesitan reconstruir una identidad de estudiantes, buscan un sentido de pertenencia, por eso son importantes los hábitos, la regularidad, la asistencia, la puntualidad. (Prof. 11. E. Educativo 1)

\subsection{SISTEMA ATENCIONAL}

El cerebro tiene circuitos especiales para cada una de sus diferentes funciones. Los circuitos de la atención están en la corteza cerebral de la zona frontal, en el área llamada prefrontal, y que controlan la memoria de trabajo, la atención, la atención y la inhibición de las respuestas (Soutullo, 2008).

La corteza prefrontal es la encargada de las funciones ejecutivas actividades como planificar una acción, iniciarla, regular si está haciendo mal o bien, darse cuenta de los errores y corregirlos, observar si se está siguiendo un plan, evitar distracciones por estímulos irrelevantes, rechazar interferencias. El déficit de atención se debe al funcionamiento cerebral subyacente y está caracterizado por una menor actividad funcional y un menor tamaño en este córtex prefrontal responsable de estas acciones.

Es importante comprender que muchos tienen problemas de atención, que no pueden estar sentados durante toda una clase, que tienen que salir a buscar "oxígeno" afuera y en vez de obligarles a estar, hay que dejar salir, que salgan y vuelvan. Lo difícil es generar la atención sostenida, ellos traen una cultura de conseguir las cosas rápido, sin sentido de proceso. Conseguir atención es difícil porque el proceso educativo no es un "continuum" porque faltan. Muchos trabajan, les cambian los horarios, no alcanzan a llegar. Vienen sin un proceso de desarrollo de hábitos, de conductas, han sido maltratados y a veces mal alimentados. La diversidad y las dificultades de mantener la atención en el aula son un factor característico y es determinante para el profesor o profesora, porque requiere ejecutar diversidad metodologías de enseñanzaaprendizaje. (Profesor 12 E. Educativo 3) 


\section{REFLEXIONES FINALES}

Avanzado el siglo XXI, el evidente avance científico y tecnológico junto a la emergencia de nuevos conflictos sociales de dimensión global, demanda una comprensión de los fenómenos educativos desde un enfoque interdisciplinar, no como la suma de respuesta desde cada disciplina, sino desde la construcción de preguntas de investigación comunes con foco en las personas en sus dimensiones biológicas y sociales. La comprensión del cerebro ayuda a derribar mitos anclados en ideas que han contribuido a segmentar a la población según capacidades, las que suelen encubrir múltiples inequidades sociales.

Para la educación de personas jóvenes y adultas la neurociencia constituye una oportunidad de revitalizar conceptos e incorporar nuevos enfoques en la comprensión de los procesos de aprendizaje y el desarrollo de propuestas pedagógicas, es una oportunidad de diálogo a partir de temas claves que requieren una mirada interdisciplinar. Las y los docentes observan a las personas que interactúan en un contexto educativo, acompañan su aprendizaje y su formación, se puede decir que observan lo tangible. La neurociencia busca comprender el cerebro sobre la base de evidencias científicas, habitualmente experimentadas en laboratorios.

La creciente centralidad que ha cobrado la neurociencia en el debate pedagógico se explica, al menos en parte, por la necesidad de contar con mayores herramientas en una profesión que cuenta con pocas certezas para actuar en un mundo complejo de acceso rápido a un volumen enorme de información y, un espacio educativo, que enfrentado a desafiantes temas valóricos y convivencia diaria.

La oportunidad consiste en abrir el diálogo a partir de temas claves buscando articular los avances científicos sobre la comprensión del cerebro con experiencias pedagógicas. Es un diálogo interdisciplinario que requiere de acercamientos sucesivos a partir de preguntas con sentido para las diferentes disciplinas.

En este diálogo la neurociencia realiza aportes que ayudan a comprender mejor las condiciones necesarias para el logro de aprendizajes y diseñar ofertas pertinentes en educación de personas jóvenes y adultas. El más importante, la evidencia que todas las personas pueden aprender a lo largo de la vida, que existe plasticidad cerebral, que el aprendizaje puede transformar el cerebro, que las neuronas tienen múltiples formas de conexión entre sí.

Los hallazgos de la neurociencia contribuyen a una mejor comprensión de las características de las y los estudiantes que participan de la educación de jóvenes y adultos. Los estudios asociados a la memoria y el recuerdo, el manejo del estrés, la organización del tiempo, la dependencia, la ansiedad y la atención favorecen que los docentes puedan contar con nuevos elementos para abordar temas claves relacionados con la motivación al aprendizaje, con la regularidad y permanencia de las personas en el proceso educativo, con el manejo de la frustración y superación de obstáculos cognitivos. Al mismo tiempo colocan el gran desafío de crear un ambiente enriquecido de aprendizaje que, reconociendo la diversidad, incluya y potencie a todas las personas que participan del proceso educativo.

\section{REFERENCIAS BIBLIOGRÁFICAS}

Elbers M. (2015). Alimentación para la neurogénesis. Revista Mente y cerebro, (74), 62-69.

Hensch, T. (2016). El poder del cerebro infantil. Revista Investigación y Ciencia, (475), 44-49. 
Jensen, E. (2010). Cerebro y aprendizaje: competencias e implicancias educativas. Ed. Narcea. Madrid (España).

López, J. C. (2012). Sinapsis para recordar. Revista Mente y Cerebro, (54), 42- 54.

Moser, May-Britt y Edvard I. Moser. (2016). El GPS del cerebro. Revista Investigación y Ciencia, (474), 17-23.

OECD (2003). La comprensión del cerebro. Hacia una nueva ciencia del aprendizaje. Editorial Santillana. Madrid (España).

Scholz, J. y Klein, M. (2011). El aprendizaje transforma el cerebro. Revista Mente y Cerebro, (51), $10-15$.

Soutullo Esperón, C. (2008). Convivir con Niños y Adolescentes con Trastorno por Déficit de Atención e Hiperactividad (TDAH). Editorial Médica Panamericana, Madrid (España).

UNESCO (2009). Conferencia Internacional de Educación de Adultos (CONFINTEA), la última, realizada el año 2009 marca la transición hacia este nuevo enfoque "De la alfabetización al aprendizaje a lo largo de la vida". Recuperado en https://unesdoc.unesco.org/search/NEXPLORE-e7b8350a-3012-4df0-9654-c78dbefd3f52

Wolf, Claudia C. (2013). Sistema de recompensa. Revista Mente y Cerebro, (61), 80-84.

Zárate, S., Cardenas Parra, F., Acevedo-Triana, C., Sarmiento-Bolaños, M. J. \& León, L. A. (2014). Efectos del estrés sobre los procesos de plasticidad y neurogénesis: una revisión. Universitas Psychologica, 13(3), 1181-1214. 